\title{
Effects of Carbon Ion Beam-induced Mutagenesis for the Screening of RED Production-deficient Mutants of Streptomyces Coelicolor JCM4020
}

\section{Masaomi Yanagisawa}

The University of Tokyo

Shumpei Asamizu ( $\nabla$ asamizu@g.ecc.u-tokyo.ac.jp )

The University of Tokyo

Katsuya Satoh

Takasaki Advanced Radiation Research Institute, National Institutes for Quantum Science and

Technology

Yutaka Oono

Takasaki Advanced Radiation Research Institute, National Institutes for Quantum Science and Technology

Hiroyasu Onaka

The University of Tokyo

\section{Research Article}

Keywords:

Posted Date: January 27th, 2022

DOI: https://doi.org/10.21203/rs.3.rs-1194810/v1

License: (9) This work is licensed under a Creative Commons Attribution 4.0 International License. Read Full License 


\section{Abstract}

Streptomyces lividans TK23 interacts with mycolic acid-containing bacteria (MACB), such as Tsukamurella pulmonis TP-B0596, and this direct cell contact activates its secondary metabolism (e.g., the production of undecylprodigiosin: RED). Here, we employed carbon $\left({ }^{12} \mathrm{C}^{5+}\right)$ ion beam-induced mutagenesis to investigate the signature of induced point mutations and further identify the gene(s) responsible for the production of secondary metabolites induced by T. pulmonis. We irradiated spores of the Streptomyces coelicolor strain JCM4020 with carbon ions to generate a mutant library. We screened the RED production-deficient mutants of S. coelicolor by mixing them with T. pulmonis TP-B0596 on agar plates, identifying the red/white phenotype of the growing colonies. Through this process, we selected 59 RED-deficient mutants from around 152,000 tested spores. We resequenced the genomes of 16 mutants and identified 44 point mutations induced by irradiation. The mutation signature of the ${ }^{12} \mathrm{C}^{5+}$-irradiated samples differed from those of the UV-irradiated, NTG-treated, or reactive oxygen species-treated samples. Via gene complementation experiments, we also revealed that two genes-glutamate synthase $(g / t B)$ and elongation factor $\mathrm{G}(f u s A)$-are responsible for the reduced production of RED.

\section{Introduction}

Streptomyces spp. is an industrially important Gram-positive filamentous actinobacterium that produces bioactive natural products (secondary metabolites) used as antibiotics, pesticides, and for anticancer treatments. ${ }^{1,2}$ Each strain contains more than 20-40 putative biosynthetic gene clusters for secondary metabolites. ${ }^{3,4}$ Although they are considered to have the potential to produce those genome-encoded cryptic secondary metabolites, the number of metabolites detectable in general laboratory culture conditions is still limited. ${ }^{4,5}$

We previously reported that a group of mycolic acid-containing bacteria (MACB; e.g., Tsukamurella pulmonis TP-B0596) affected the secondary metabolism of actinomycetes in combined-culture. ${ }^{6}$ Combined-culture is a co-culture method that involves the combination of actinomycetes and MACB to activate the production of secondary metabolites by actinomycetes. MACB are also a group of actinomycetes containing specific long-chain fatty acids $\left(\mathrm{C}_{30}-\mathrm{C}_{60}\right.$ mycolic acids) on the cell wall. 7,8 MACB (e.g., T. pulmonis TP-B0596) can activate the production of undecylprodigiosin (RED) and actinorhodin (ACT) by Streptomyces lividans TK23, which are not produced by this strain under general laboratory culture conditions. ${ }^{6}$ In fact, T. pulmonis TP-B0596 was shown to be effective at inducing the production of a diverse range of secondary metabolites by various actinomycetes, ${ }^{9-17}$ and this strategy was also found to be efficient for enhancing production during heterologous expression. ${ }^{18-20}$

Studies have reported that the above-mentioned activation of RED and ACT did not occur through the provision of culture extracts or killed bacteria. ${ }^{6,21}$ However, scanning electron microscopy (SEM) revealed that S. lividans and several MACB formed co-aggregates. ${ }^{21}$ Meanwhile, co-aggregation and the production of RED and ACT were not observed upon the addition of killed MACB (e.g., killed by $\mathrm{y}$-ray 
irradiation or formaldehyde fixation). ${ }^{21}$ Therefore, it was suggested that the formation of co-aggregates generates continuous physical cell contact with living T. pulmonis, leading to activation of the production of RED and ACT by S. lividans.

A convenient strategy for bacterial mutagenesis is ultraviolet (UV) irradiation or chemical treatment with $N$-methyl- $N$-nitro- $N$-nitrosoguanidine (NTG). UV radiation is an ubiquitous and potent DNA-damaging mutagen. ${ }^{22}$ The most common type of DNA damage caused by UV is covalent linkages between two adjacent pyrimidines, resulting in the generation of a cyclobutane pyrimidine dimer (CPD) and pyrimidine(6-4)pyrimidone photoproducts (6-4PP). ${ }^{23}$ Failure to detect and repair such DNA lesions is a major cause of mutagenesis. This chemical coupling results in the mutation signature of C-to-T transitions. ${ }^{22} \mathrm{UV}$ is also involved in the production of reactive oxygen species (ROS) from cellular $\mathrm{O}_{2}$, which has been reported to be generated by activating cellular substances, such as riboflavin, tryptophan, and porphyrin. ${ }^{22}$ ROS attack DNA and produce the mutagenic 8-oxo-7,8-dihydroguanine (8-oxoG). ${ }^{24}$ The signature of ROS-induced mutation is C-to-A transversion, ${ }^{24}$ which is less common but also present in the genomes of cells mutated by UV irradiation. ${ }^{22,25}$ NTG induces mutations by alkylating purines and pyrimidines in DNA. The majority of mutations caused by NTG are C-to-T transitions, a signature similar to that of UV-induced mutations. ${ }^{26}$ Although UV and NTG are convenient and widely used mutagens, their signatures can cause a bias that limits the ability to generate random mutations evenly across the whole of the bacterial genome.

lonizing radiation induces DNA double-strand breaks (DSBs), a particularly serious form of DNA damage that is especially deleterious to cells. Its mutagenic effects depend on high linear energy transfer (LET). Ion beams have been used for plant breeding and shown to have highly lethal and mutagenic effects, causing a low number of mutations in a locus but instead large-scale genomic variations. ${ }^{27}$ Recently, the use of ion beam mutagenesis technology has been expanded to the breeding of various microorganisms. ${ }^{28,29}$ Genomic analyses of the ion beam-induced mutants have been reported, clarifying the effects of ion beam mutagenesis on microorganisms such as Escherichia coli ${ }^{30}$, Bacillus subtilis $^{31}$, and Saccharomyces cerevisiae ${ }^{32}$. It has been proposed that radiation doses that leave a surviving fraction of a population of $1-10 \%$ are effective for achieving mutagenesis in microorganisms. ${ }^{33}$ Regarding the application of heavy-ion mutagenesis to Streptomyces spp., several examples that show the improved production of useful secondary metabolites have been reported. ${ }^{33-35}$ However, because the number of applications using heavy-ion mutagenesis for bacteria is still limited, and the mutants have not been comprehensively characterized at the genome scale, there is still a need to understand the signature of mutations in the Streptomyces genome. ${ }^{28,29}$

In this study, we performed mutagenesis using carbon ions $\left({ }^{12} \mathrm{C}^{5+}\right)$ on Streptomyces coelicolor JCM4020 and screened RED-deficient mutants under conditions where this strain could interact with T. pulmonis TP-B0596. The ${ }^{12} \mathrm{C}^{5+}$ ion beams (surface LET, $107 \mathrm{keV} / \mu \mathrm{m}$ ) were highly mutagenic among the different levels of LET for the breeding of microorganisms, so we selected this ion to obtain mutants of interest. 
Using T. pulmonis TP-B0596 as an inducer of RED production, mutants of Streptomyces coelicolor JCM4020 that exhibited the loss or reduction of RED production were screened from a pooled spore mutant library. We further performed resequencing of the mutant genome from 16 randomly selected mutants using the Illumina MiSeq system. The sites of DNA mutation were identified and two genes were found to be responsible for the reduced production of RED by gene complementation study.

\section{Materials And Methods}

\section{Preparation of mutant library using carbon ion beams}

Streptomyces coelicolor JCM4020 was used as a reference and its whole-genome nucleotide sequence was deposited in a public database with accession number (submitted and waiting for approvement) (BioProject Accession: PRJDB12707, BioSample Accession SAMD00433952). A total of $1 \times 10^{8}$ spores of JCM4020 were transferred to a $1.5 \mathrm{ml}$ tube from the freeze stock and $900 \mu \mathrm{l}$ of $0.5 \times$ PBS with $20 \%$ glycerol was added. After centrifugation $\left(3,000 \mathrm{rpm}, 3 \mathrm{~min}, 4^{\circ} \mathrm{C}\right)$, the supernatant was discarded and $1 \mathrm{ml}$ of $0.1 \times$ PBS was added to suspend the spores, followed by the application of $0.5 \mathrm{ml}$ of sample on a mixed cellulose ester membrane ( $47 \mathrm{~mm}$ i.d., $0.2 \mu \mathrm{m}$ ) on the Petri dish $(55 \mathrm{~mm}$ i.d.). After air-drying inside the flow cabinet, the Petri dish was covered by 7.5 - $\mu$ m-thick kapton polyimide film (Toray-Dupont) and ${ }^{12} \mathrm{C}^{5+}$ ions (220 MeV, surface LET: $107 \mathrm{keV} / \mu \mathrm{m}$ ) were irradiated by the AVF cyclotron at TIARA, QST, Takasaki. The sample was prepared twice. The first irradiation doses were $100 \mathrm{~Gy}, 500 \mathrm{~Gy}$, and 1,000 Gy ( $\mathrm{n}=3 \mathrm{each}$ ), and the second irradiation doses were $10 \mathrm{~Gy}, 50 \mathrm{~Gy}, 100 \mathrm{~Gy}$, and $200 \mathrm{~Gy}$ ( $\mathrm{n}=3 \mathrm{each}$ ). The irradiated spores were recovered by adding $0.5 \mathrm{ml}$ of $20 \%$ glycerol solution and scraping the surface with a spoon. This step was repeated four times and the suspended spore solution was collected and centrifuged $(3,000$ $\mathrm{rpm}, 5 \mathrm{~min}, 4^{\circ} \mathrm{C}$ ). After discarding the supernatant, $1 \mathrm{ml}$ of $20 \%$ glycerol solution was added and the sample was stored at $-80^{\circ} \mathrm{C}$ as the irradiated spore stock until use.

\section{Survival rates and aerial mycelium formation rates}

The irradiated spore stock was further diluted as appropriate using sterilized $\mathrm{H}_{2} \mathrm{O}$ and $100 \mu \mathrm{l}$ was applied on a tryptone soya broth agar plate. After incubation at $30^{\circ} \mathrm{C}$ for 3 days, the number of colony forming units (CFU) was counted. The survival rate was calculated by comparison to the 0 Gy sample, which was similarly treated but without irradiation. To obtain the rate of aerial mycelium formation, approximately 100 colonies were grown on each MS agar ${ }^{49}$ plate and incubated at $30^{\circ} \mathrm{C}$ for 7 days. Colonies forming aerial mycelium were counted and divided by the total number of inoculated colonies.

\section{Screening of mutants}

Initial screening of the mutants was performed in two ways. (Supplemental Figure 1 and 2) For the first one, the cell stock of T. pulmonis TP-B0596 was diluted in sterilized $\mathrm{H}_{2} \mathrm{O}$ and $100 \mu$ of this cell (about $1 \times$ $10^{5}$ cells) suspension was applied to YGGS agar. ${ }^{21}$ On the same plate, an irradiated spore stock was diluted to give approximately 100 colonies on a single agar plate and incubated at $30^{\circ} \mathrm{C}$ for $4-7$ days. $T$. pulmonis grew in the bacterial lawn, where $S$. coelicolor colonies could also be found. Most of the $S$. 
coelicolor colonies were red in color, but colonies with weak or no coloring were selected. The T. pulmonis cells were removed by growing each picked colony directly on mixed cellulose ester membrane $(0.45 \mu \mathrm{m})$. We used the feature that $S$. coelicolor filamentous cells can pass through a $0.45 \mu \mathrm{m}$ membrane and reach the agar medium, but T. pulmonis with its larger size cannot. After incubation at $30^{\circ} \mathrm{C}$ for 3 to 7 days, the membrane was removed and further incubated for 2-7 days. The obtained filtered mutant cells were subcultured on new YGGS agar and incubated at $30^{\circ} \mathrm{C}$ for $3-5$ days. The formed colonies were suspended in $20 \%$ glycerol solution and stored at $-80^{\circ} \mathrm{C}$ until use.

In the second screening approach, we considered the possibility that irradiated spores contain damaged DNA and thus require a period of recovery to form a colony from a single spore. Growth with T. pulmonis from the beginning may thus affect the growth of the mutants and thus reduce their yield. On the YGGS agar plate, irradiated spore stock was applied to have approximately 60 colonies on a single agar plate and incubated at $30^{\circ} \mathrm{C}$ for 3 days. On the other YGGS plate, T. pulmonis was grown in the bacterial lawn by incubation at $30^{\circ} \mathrm{C}$ for 3 days. A metal cylinder covered by velour-surfaced material was used to stamp the agar culture of T. pulmonis and then stamped on the agar culture of $S$. coelicolor to inoculate T. pulmonis (similar to traditional colony hybridization in the molecular biology technique). After incubation at $30^{\circ} \mathrm{C}$ for 3 days, mutant colonies of $S$. coelicolor were picked and further isolated by the same methods as described above.

\section{Mutant phenotype examination under different culture conditions}

The phenotype of the mutants obtained by the first screening was first confirmed by dual culture with $T$. pulmonis. Two microliters of T. pulmonis cell stock was spotted on the agar plate and $2 \mu \mathrm{l}$ of $S$. coelicolor mutant cell stock was spotted at a distance of $1 \mathrm{~cm}$. The cells were incubated at $30^{\circ} \mathrm{C}$ and the production of RED in the area where the colonies contacted each other was visually examined in comparison to the phenotype of the wild-type strain as a control. The mutant with less or no productivity of RED was further grown on minimal medium ${ }^{49}$ by spotting $2 \mu$ of $S$. coelicolor mutant cell stock; the growth was then visually examined after incubation at $30^{\circ} \mathrm{C}$ for 7 days. The mutant that could grow on the minimal medium was further tested for the ability to form aerial mycelium. Two microliters of $S$. coelicolor mutant cell stock was used to inoculate Bennett's maltose agar ${ }^{49}$ and MS agar medium ${ }^{49}$, followed by incubation at $30^{\circ} \mathrm{C}$ for 7 days. A visible white appearance of the colony was used to judge whether or not aerial mycelium had formed. The mutant forming aerial mycelium was further tested for the ability to produce RED under $\mathrm{NaCl}$ stress. Two microliters of $S$. coelicolor mutant cell stock was used to inoculate Bennett's maltose with $1 \% \mathrm{NaCl}$. Production of RED under osmotic stress was examined by the visible red appearance of the colony.

\section{PacBio RSIl sequencing of wild type, DNA library preparation, and MiSeq sequencing of mutants}


Genomic DNA of the wild type and mutants was isolated by the CTAB protocol. ${ }^{49}$ Single-molecule realtime sequencing (PacBio RSII) was used to determine the complete genome sequence of $S$. coelicolor JCM4020 by BGI (https://www.bgi.com). A library was prepared twice to generate $20 \mathrm{~kb}$ fragments and 0.3 and $0.9 \mathrm{~Gb}$ of sequence data were obtained. The obtained long read sequence data were assembled by Canu/Celera Genome Assembler ${ }^{50}$ and a single contig was obtained. The CDS were deduced using the DFAST (https://dfast.ddbj.nig.ac.jp/) pipeline with the automatic annotation of gene function. ${ }^{51}$ Briefly, 4671 CDS features were detected by MetaGeneAnnotator ${ }^{52}, 54$ tRNA features were detected by Aragorn, ${ }^{53}$ 6 rRNA features were detected by Barrnap (https://github.com/tseemann/barrnap).

The genomes purified from the mutants and wild type were broken up into approximately $300 \mathrm{bp}$ fragments by NEBNext ${ }^{\circledR}$ dsDNA Fragmentase ${ }^{\circledR}$. Agencourt AMPure XP (Beckman Coulter) was then used to purify these DNA fragments. Agilent Bioanalyzer 2100 with the High Sensitivity DNA kit (Agilent) was used to confirm the fragment size. The DNA library was prepared using NEBNext@ Ultra ${ }^{\text {TM }}$ II DNA Library Prep Kit for Illumina ${ }^{\circledR}$. Index primers were selected from NEBNext ${ }^{\circledR}$ Multiplex Oligos for Illumina ${ }^{\circledR}$ (Index Primers Sets 1 and 2, NEB \#E7335, \#E7500). The purity and yield of the generated DNA library were analyzed by Agilent Bioanalyzer 2100 with High Sensitivity DNA kit. MiSEQ (Illumina) with MiSeq Reagent Kit v3 (paired end, $75 \times 2$ cycles) was used for sequencing. The obtained short-read FASTA data were imported and analyzed using CLC Genomic Workbench software ver. 10 (Qiagen). After mapping the short reads to the reference genome sequences of JCM4020 wild type, nucleotide substitutions, insertions, and deletions were detected by comparison to the MiSEQ sequence data of the wild type obtained at the same time. Large deletions of the genome were searched manually. The identified point mutations were confirmed by Sanger sequencing of the PCR-amplified products.

\section{Gene complementation study}

Using genomic DNA of the JCM4020 strain as a template, primers (Supplemental Table 2) were used to amplify the DNA fragments containing gltB (sco2026 homolog) and fusA (sco4661 homolog) by PCR. To consider the expression level in the original strain, the original promoter was used to express the gene. The cloning region containing the 5'-UTR of the genes was determined based on prediction of the polycistronic transcription unit using our RNA-sequencing data (data not shown). KOD Plus NEO (Toyobo) was used for the PCR, following the manufacturer's protocol. The amplified $6.46 \mathrm{~kb}$ DNA fragment containing the region homologous to sco2026-25 was digested by $\mathrm{BamHI}$ and HindIII, and ligated into the corresponding site of the PTYM19t plasmid ${ }^{54}$ to generate PTYM19-gltB. The amplified $4.64 \mathrm{~kb}$ DNA fragment containing the region homologous to sco4659-62 was digested by EcoRI and HindIII, and ligated into the corresponding site of the pTYM19t plasmid to generate pTYM19-fusA. The standard protocol for a conjugation method using E. coli ET12567 (pUZ8002) was performed to introduce the plasmids into the spores of the JCM4020 strain. ${ }^{49}$ The obtained mutants were selected using $20 \mu \mathrm{g} / \mathrm{ml}$ thiostrepton and applied for further study. To compare the responses among the wild type, mutant, and gene-complemented mutant, dual culture with T. pulmonis was performed on an agar plate. Spore stock solutions ( $\left.2 \mu \mathrm{L}, 2-3 \times 10^{5} \mathrm{CFU}\right)$ of mutant harboring empty vector (pTYM19t), mutant harboring the expression plasmid (pTYM19t-gltB), and JCM4020 wild type harboring empty vector 
(pTYM19t) were inoculated by spotting on YGGS agar medium. At the same time, cell stock solution (2 $\mu \mathrm{L}, 5 \times 10^{6} \mathrm{CFU}$ ) of $T$. pulmonis was spotted on the agar medium. After the inoculation, the cells were grown at $30^{\circ} \mathrm{C}$ for 7 days and observed over time. The same procedure was carried out for the gene complementation of fusA.

\section{Results}

\section{Genome sequence of Streptomyces coelicolor JCM4020}

The genome size of $S$. coelicolor JCM4020 was 8,634,640 bp, comprising a single linear chromosome. JCM4020 did not contain any plasmids. Its GC content was $72.2 \mathrm{~mol} \%$ and the number of coding sequences (CDS) was 7799. The identified CDS were almost identical to those of the model actinomycetes strain S. coelicolorA3(2) M145, which has a genome size of 8,667,507 bp, $72.1 \mathrm{~mol} \% \mathrm{GC}$ content, and $7877 \mathrm{CDS} .{ }^{36}$ Complete comparison of CDSs using Multi BLAST between two strains is shown in Supplemental File 1. In this paper, the SCO numbers used for S. coelicolor A3(2) M145 gene and CDS designations are used for convenience. S. coelicolor JCM4020 was used in this experiment since the strain does not produce RED or ACT in monoculture conditions. Secondary metabolite gene clusters in the strain JCM4020 were predicted by antiSMASH ${ }^{37}$ (Supplemental Figure 1).

${ }^{12} \mathrm{C}^{5+}$ ion irradiation and dose-dependent survival rates

Mutagenesis by carbon ion beams was performed at TIARA (Takasaki lon Accelerators for Advanced Radiation Application), QST (National Institutes for Quantum Science and Technology). First, 100 Gy, 500 Gy, and 1,000 Gy of ${ }^{12} \mathrm{C}^{5+}$ ions were irradiated. The survival rates were measured by counting the colony forming units (CFU), which were then compared with those upon no irradiation, giving values of $68.8 \pm$ $25.1 \%$ for $100 \mathrm{~Gy}, 7.4 \pm 0.68 \%$ for $500 \mathrm{~Gy}$, and $0.52 \pm 0.10 \%$ for 1,000 Gy irradiation (Table 1 ). We also tested the formation of aerial mycelium in this step using the colony morphology as an indication. Overall, $99.9 \pm 0.065 \%$ of the formed colonies generated aerial mycelium at $0 \mathrm{~Gy}, 93.6 \pm 1.2 \%$ at $100 \mathrm{~Gy}$, $63.3 \pm 4.0 \%$ at $500 \mathrm{~Gy}$, and $40.4 \pm 16.2 \%$ at $1,000 \mathrm{~Gy}$. Second, in line with the procedure used in the first

irradiation, $10 \mathrm{~Gy}, 50 \mathrm{~Gy}, 100 \mathrm{~Gy}$, and $200 \mathrm{~Gy}$ of ${ }^{12} \mathrm{C}^{5+}$ ions were irradiated. The survival rates were $55.0 \pm$ $3.5 \%$ at $10 \mathrm{~Gy}, 36.1 \pm 8.6 \%$ at $50 \mathrm{~Gy}, 36.0 \pm 5.5 \%$ at $100 \mathrm{~Gy}$, and $13.0 \pm 4.1 \%$ at $200 \mathrm{~Gy}$, which were relatively consistent with the rates after the first irradiation (Table 1). The formation of aerial mycelium was also tested, showing rates of $99.9 \pm 0.065 \%$ for $0 \mathrm{~Gy}, 99.3 \pm 0.35 \%$ for $10 \mathrm{~Gy}, 99.3 \pm 0.43 \%$ for $50 \mathrm{~Gy}$, $98.4 \pm 1.1 \%$ for $100 \mathrm{~Gy}$, and $90.6 \pm 2.3 \%$ for $200 \mathrm{~Gy}$. Overall, the survival rate and aerial mycelium formation rate showed a certain correlation with the irradiation dose.

\section{Screening of RED production-deficient mutants}

We chose mutant spore libraries from the first 100 Gy irradiation, second 100 Gy irradiation, and second $200 \mathrm{~Gy}$ irradiation for the screening of mutants, considering the survival rates. In total, we tested approximately 152,000 spores (based on estimation from the CFU) and selected 118 mutants (with a 
yield of $0.078 \%$ ) that showed lower or lost production of RED upon incubation with T. pulmonis TP-B0596 (Figure 1, Supplemental Figure 1 and 2). The low yield (0.078\%) of mutant may have been because we used the mutant spore library showing a relatively high survival rate (13.0-68.8\%). Radiation doses

giving a surviving fraction of $1-10 \%$ have been proposed for effective mutagenesis in microorganisms. ${ }^{33}$ The use of a sample with a lower survival rate may increase the number of mutants exhibiting abnormal RED production, but it may also increase multiple undesired mutations in the genome, leading to difficulty identifying the genes responsible for the phenotype. We then tested the growth on minimal medium and the formation of aerial mycelium for 118 mutants, 59 of which showed no deficiency (Figure 1). We excluded mutants that could not grow on the minimal medium from further analysis because they may exhibit specific auxotrophy by possessing a primary metabolism-related mutation that significantly affects growth, in turn affecting the secondary metabolism. We also excluded mutants that could not form aerial mycelium from further analysis at this point because the genes responsible for the bald phenotype have been extensively studied ${ }^{38,39}$ and to reduce the rediscovery of already-characterized genes. There was a concern that the mutations would occur in the biosynthetic gene cluster of RED, which directly affect the production. The production of RED by Streptomyces coelicolorA3(2) was reported to be activated by the addition of $\mathrm{NaCl}^{40}$ We then tested the effect of salt stress by adding $1 \%$ $\mathrm{NaCl}$ to the medium because $S$. coelicolor JCM4020 did not produce RED on the tested normal medium in monoculture. Five mutants showed RED production comparable to that of the wild type in the saltstressed condition (Figure 1 and 2). Although the mechanism of its induction by salt stress was unclear, it was confirmed that at least these mutants do not contain significant lesions in the biosynthetic gene cluster of RED.

\section{Identified point mutations}

Subsequently, we performed genome re-sequencing to identify the point mutations in the genome of screened mutants to investigate the signature of induced point mutations and further identify the gene(s) responsible for the production of secondary metabolites. Overall, 16 mutants were selected, namely, 3 mutants that produce RED in salt stress condition and 13 mutants that form aerial mycelium, which showed a consistent phenotype (Figure 1, Supplemental Figure 3). The genome was resequenced using a next-generation sequencer, MiSEQ (Illumina). Upon comparison with the sequence of the wild type, we identified 58 point mutations (Table 2 and 3). The identified point mutations were confirmed by further Sanger sequencing. Six mutants (Mt 202001, Mt 202004, Mt 202007, Mt 205011, Mt 208014, Mt 20980) contained an identical C-to-A transversion at genomic position 4,593,233 bp and the insertion of a $\mathrm{G}$ at genomic position 4,565,012 bp (Table 2). Moreover, two mutants (Mt 209010, Mt 203013) contained the insertion of a $\mathrm{C}$ at genomic position $4,564,124 \mathrm{bp}$ (Table 2). It is unlikely for an identical point mutation to be induced at the same position, so these $14(6+6+2)$ point mutations were considered to have arisen naturally during cell growth for spore preparation. Therefore, the other 44 point mutations were considered to have been induced by the carbon ions. Upon $100 \mathrm{~Gy}$ of irradiation, 14 point mutations from 7 mutants (average 2.0 point mutations/mutant) were found, while upon $200 \mathrm{~Gy}$ of irradiation, 30 point mutations from 9 mutants (average 3.3 point mutations/mutant) were found. The number of mutations showed a certain correlation with the irradiation dose. Among these 44 mutations, there were 31 base 
substitutions, 5 insertions, and 8 deletions (Table 3). The 44 point mutations were distributed relatively evenly across the whole genome. Meanwhile, we did not detect any large-scale genomic variations, such as large deletions, translocations, or inversions in the carbon ion-irradiated S. coelicolor JCM4020.

\section{Identification of amino acid mutations}

Amino acid mutations in the CDS caused by point mutations were identified. Among the identified 44 point mutations, at the amino acid level they caused 13 missense mutations, 2 nonsense mutations, 9 frameshifts, 1 amino acid insertion, 2 amino acid deletions, and 7 silent mutations, while the remaining 10 were in noncoding regions (Table 2). Overall, 27 amino acid mutations were considered to affect the function of the encoded protein. Two mutants (Mt 106003, Mt 201001) contained mutations in RED biosynthetic genes (redH and redP, respectively), indicating that these mutations directly cause deficiency of RED biosynthesis (Table 2, Supplemental Figure 3_5). As described previously, naturally arising point mutations involving the insertion of a $G$ at $4,565,012$ bp in six mutants and the insertion of a $C$ at $4,564,124$ bp in two mutants cause frameshift in the SarA (SC04069) homolog originally found in Streptomyces coelicolor A3(2). ${ }^{41}$ As the deletion of sarA causes a defect in RED production ${ }^{41}$, it was considered that sarA mutants were accumulated by our screening method. Finally, six mutants containing a total of 14 amino acid mutations were considered for the identification of candidate genes involved in the production of RED induced by T. pulmonis stimulation.

\section{Gene complementation for phenotypic recovery}

Three of the 16 genome-resequenced mutants (Mt 108013, 203010, Mt 107004) formed aerial mycelium and produced RED in agar medium containing $1 \% \mathrm{NaCl}$ in Bennett's maltose at levels comparable to those in the wild type (Figures 2, Supplemental Figure 3_1). We performed gene complementation for Mt 108013 and Mt 203010. Mt 108013 possessed a mutation in glutamine synthase (GltB, SCO2026 homolog), and Mt 203010 possessed one in elongation factor G (EF-G) (FusA, SC04661 homolog) (Table 2). In Mt $108013, C^{597}$ of the $g / t B$ gene was deleted, resulting in frameshift of the amino acid sequence (Supplemental Figure 4). The DNA fragment containing the glt $B$ gene was amplified by PCR from the wild-type JCM4020 and cloned into the PTYM19t vector. The pTYM19t-gltB vector was introduced into Mt 108013 and the production of RED was compared with that of the wild type. Mt 108013 complemented by PTYM19-gltB recovered the capacity to produce RED in combined-culture with $T$. pulmonis, compared with Mt 108013 with an empty vector (Figure 3, Supplemental Figure 6). In Mt 203010, $C^{147}$ of the fusA gene was deleted, which also resulted in frameshift of the amino acid sequence. (Supplemental Figure 5) The DNA fragment containing the fusA gene with 5'-UTR was amplified by PCR from the wild-type JCM4020 and cloned into the pTYM19t vector. The pTYM19t-fusA vector was introduced into Mt 203010 and its production of RED was compared with that of the wild type. Mt 203010 complemented by PTYM19-fusA recovered the production of RED in combined-culture with T. pulmonis, compared with Mt 203010 with an empty vector (Figure 3, Supplemental Figure 7).

\section{Discussion}


In this study, we evaluated the mutagenesis signature generated in Streptomyces spp. by a carbon ion beam. To screen the mutants, samples irradiated with 100 and 200 Gy were chosen based on the associated survival rates. Overall, the survival rate was dose-dependent, but showed some variation between the first and second irradiations. The ${ }^{12} \mathrm{C}^{5+}$ ion beam can transfer energy to samples according to the Bragg curve. On the sample surface, the level of irradiation was $107 \mathrm{keV} / \mu \mathrm{m}$ and the Bragg peak gave the highest energy (more than $600 \mathrm{keV} / \mu \mathrm{m}$ ) which is right before the ion progression terminates. The penetration range of ${ }^{12} \mathrm{C}^{5+}$ ion in water is $1110 \mu \mathrm{m}$. Therefore, it was considered that the inconsistent thickness of the sample (overlapping spores in this case) may have affected the LET between the sample and thus affected the survival rate. ${ }^{29}$

By resequencing the genomes of 16 mutants, we found 58 point mutations. Fourteen of those were predicted to be naturally arising mutations. Therefore, 44 mutations were predicted to have been induced by the ion beam, which included 18 transitions, 13 transversions, 5 insertions, and 8 deletions. Among the 31 nucleotide substitutions found in this study, 13 mutations $(41.9 \%, 13 / 31)$ were G/C-to-A/T transitions (Table 3). This relatively low frequency of G/C-to-A/T transitions gave a different point mutation ratio overall from the pattern of UV irradiation (usually gave more than $70 \%$ ). Five other $A / T-t o-G / C$ transitions $(16.1 \%, 5 / 31)$ formed a signature not known to be produced by other methods of mutagenesis. For the other mutations, $13(41.9 \%, 13 / 31)$ were transversions, involving a change from a purine to a pyrimidine base or vice versa (Table 3). Among these 13 transversions, $9(29.0 \%, 9 / 31)$ were C/G-to-A/T mutations and 4 were $\mathrm{G} / \mathrm{C}$-to-C/G mutations. $\mathrm{G}$ to $\mathrm{T}$ is a signature point mutation induced by reactive oxygen species (ROS). ROS can be generated by UV irradiation, as well as heavy-ion irradiation. ${ }^{42}$ The results indicate that the majority of mutation types $[71.0 \%,(13+9) / 31]$ are similar to the mutations induced by UV ( $C$ to $\mathrm{T}$ ) or ROS ( $\mathrm{C}$ to $\mathrm{A}$ ). Additionally, when we mapped the 44 point mutations on the genome, the distribution was found to be relatively even across the whole genome. Theoretically, a carbon ion beam can attack DNA and introduce double-strand breaks. The capacity to repair such breaks might be less effective in bacteria, although in Streptomyces coelicolor A3(2) Ku protein (SC05309) was shown to be responsible for non-homologous end joining (NHEJ). ${ }^{43}$ In addition to SNPs, we found that $29.5 \%(13 / 44)$ of the mutations were indels, including five insertions and eight deletions. Because there has been little study of the effects of carbon ions on mutagenesis in Streptomyces bacteria, further accumulation of findings may be needed to reveal the detailed signature of carbon ion mutagenesis. Taken together, our findings indicate specific features of mutagenesis produced by irradiation with carbon ions, involving a different mutational signature from those in conventional mutagenesis methods.

Using the screening methods applied in this study, we hoped to obtain mutants with mutations in genes involved in the response to and/or regulation of the stimulation provided by T. pulmonis. As described above, naturally occurring point mutations involving the insertion of a $G$ at $4,565,012$ bp in six mutants and the insertion of a C at 4,564,124 bp in two mutants caused frameshift in the SarA (SC04069) homolog. ${ }^{41}$ The sarA (sco4069) gene product is annotated as a hypothetical membrane protein and was also found in a transposon mutagenesis study, which showed that its inactivation had negative effects on the production of RED and actinorhodin. ${ }^{44,45}$ Therefore, it was suggested that the RED production 
deficiency of the eight mutants had been caused by inactivation of the sarA gene. Although the function of SarA in the production of RED and ACT is unknown, it was demonstrated that interaction with $T$. pulmonis also cannot recover this production and suggested that SarA may be involved in the common regulatory mechanism, leading to the production of RED upon T. pulmonis stimulation. The gltB gene was found to be responsible for the phenotype of reduced production of RED. GItB is part of GOGAT, a conserved primary metabolism enzyme that generates two molecules of glutamate from glutamine and 2-oxoglutarate. ${ }^{46}$ Glutamate plays a central role in nitrogen metabolism, including the synthesis of proline or serine that become direct precursors for the biosynthesis of RED. ${ }^{47}$ Therefore it was speculated that limitation of the precursor supply caused the reduced production of RED associated with $g / t B$ mutation. In addition to the above findings, the fusA gene was found to be responsible for the phenotype of reduced RED production. The fusA gene encodes EF-G, which is widely conserved in organisms and an essential factor for ribosome translocation. ${ }^{48} \mathrm{~S}$. coelicolorpossesses an ortholog of the fusA gene (fusB gene; sco6589 or sco1528), which might complement the function of FusA, resulting in the mutation not being lethal to the cell. It was speculated that deletion of the principal EF-G (fusA) in Mt 203010 may delay translation and therefore affect the production of RED. In Mt 107004, deletion of CGA (486-488) in the $\mathrm{rp} / 4$ gene was observed. As a factor responsible for ribosome translation, fusA causes reduced RED production, and the mutation in this ribosomal protein was also considered to be involved in the REDdeficient phenotype by affecting its translation. Considering the functions of GItB and FusA, they may not have direct relationships with the system regulating the response to T. pulmonis stimulation that leads to RED biosynthesis, but it was considered that the disturbance of essential metabolism associated with their mutations can alter the production of RED. In addition to the gltB and fus $A$ genes, sarA that is involved in the production of RED was rediscovered. As mutations in these genes reduced the basal production of RED in monoculture, it was confirmed that these genes are indeed responsible for the basal production of RED, but again they may not be relevant to the response to and/or regulatory mechanism of T. pulmonis stimulation. However, we have not examined all of the mutations by complementation to identify the genes involved in the observed phenotype of reduced or lost production of RED. Further identification of other mutated genes may provide new insight into the mechanism behind the production of RED, and deepen our understanding of the responses to and/or regulatory mechanisms of the stimulation by MACB, including T. pulmonis TP-B0596.

\section{Declarations}

\section{Acknowledgments:}

$\mathrm{SA}, \mathrm{KS}$, and $\mathrm{HO}$ designed the research. MY performed mutant screening and construction of plasmids. SA performed genome analysis, resequencing, and gene complementation. KS and YO performed carbon-ion beam irradiation. All authors analyzed the data. SA wrote the main text and prepared figures and tables. $\mathrm{HO}, \mathrm{YO}$, and KS reviewed the manuscript. We thank Prof. Yasuo Ohnishi and Dr. Takeaki Tezuka for helpful discussions on the results of this study. E. coli ET12567 (pUZ8002) was a kind gift from Prof. Mervin Bibb at John Innes Centre (UK). We also thank Edanz (https://jp.edanz.com/ac) for editing a draft 
of this manuscript. This study was supported by a Grant-in-Aid from the Institute for Fermentation, Osaka (IFO), and Amano Enzyme Foundation. This study was also partially supported by KAKENHI research grants (16K18673 to SA and $18 \mathrm{H} 02120$ to $\mathrm{HO}$ ), an IFO general research grant from IFO (to SA), and the JSPS A3 Foresight Program (to $\mathrm{HO}$ and SA). All authors declare no conflict of interest associated with this manuscript.

\section{References}

1. Newman, D. J. \& Cragg, G. M. Natural Products as Sources of New Drugs over the Nearly Four Decades from 01/1981 to 09/2019. J Nat Prod 83, 770-803, doi:10.1021/acs.jnatprod.9b01285 (2020).

2. Asamizu, S. Biosynthesis of nitrogen-containing natural products, $\mathrm{C7N}$ aminocyclitols and bisindoles, from actinomycetes. Biosci Biotech Bioch 81, 871-881, doi:10.1080/09168451.2017.1281726 (2017).

3. Navarro-Munoz, J. C. et al. A computational framework to explore large-scale biosynthetic diversity. Nat Chem Biol 16, 60-+, doi:10.1038/s41589-019-0400-9 (2020).

4. Nett, M., Ikeda, H. \& Moore, B. S. Genomic basis for natural product biosynthetic diversity in the actinomycetes. Nat Prod Rep 26, 1362-1384, doi:10.1039/b817069j (2009).

5. van Bergeijk, D. A., Terlouw, B. R., Medema, M. H. \& van Wezel, G. P. Ecology and genomics of Actinobacteria: new concepts for natural product discovery. Nat Rev Microbio/ 18, 546-558, doi:10.1038/s41579-020-0379-y (2020).

6. Onaka, H., Mori, Y., Igarashi, Y. \& Furumai, T. Mycolic acid-containing bacteria induce natural-product biosynthesis in Streptomyces species. Appl Environ Microbiol 77, 400-406, doi:10.1128/AEM.0133710 (2011).

7. Jankute, M., Cox, J. A. G., Harrison, J. \& Besra, G. S. Assembly of the Mycobacterial Cell Wall. Annu Rev Microbio/ 69, 405-423, doi:10.1146/annurev-micro-091014-104121 (2015).

8. Ventura, M. et al. Genomics of Actinobacteria: tracing the evolutionary history of an ancient phylum. Microbiol Mol Biol Rev 71, 495-548, doi:10.1128/MMBR.00005-07 (2007).

9. Pan, C. Q. et al. Amycolapeptins A and B, Cyclic Nonadepsipeptides Produced by Combined-culture of Amycolatopsis sp. and Tsukamurella pulmonis. J Org Chem 86, 1843-1849, doi:10.1021/acs.joc.0c02660 (2021).

10. Hoshino, S., Onaka, H. \& Abe, I. Activation of silent biosynthetic pathways and discovery of novel secondary metabolites in actinomycetes by co-culture with mycolic acid-containing bacteria. $J$ Ind Microbiol Biotechnol 46, 363-374, doi:10.1007/s10295-018-2100-y (2019).

11. Hoshino, S. et al. Catenulobactins A and B, Heterocyclic Peptides from Culturing Catenuloplanes sp. with a Mycolic Acid-Containing Bacterium. J Nat Prod 81, 2106-2110, doi:10.1021/acs.jnatprod.8b00261 (2018). 
12. Hoshino, S. et al. Mycolic Acid Containing Bacterium Stimulates Tandem Cyclization of Polyene Macrolactam in a Lake Sediment Derived Rare Actinomycete. Org Lett 19, 4992-4995, doi:10.1021/acs.orglett.7b02508 (2017).

13. Sugiyama, R. et al. Discovery and Total Synthesis of Streptoaminals: Antimicrobial [5,5]Spirohemiaminals from the Combined-Culture of Streptomyces nigrescens and Tsukamurella pulmonis. Angew Chem Int Ed Eng/ 55, 10278-10282, doi:10.1002/anie.201604126 (2016).

14. Sugiyama, R. et al. 5-Alkyl-1,2,3,4-tetrahydroquinolines, new membrane-interacting lipophilic metabolites produced by combined culture of Streptomyces nigrescens and Tsukamurella pulmonis. Org Lett 17, 1918-1921, doi:10.1021/acs.orglett.5b00607 (2015).

15. Hoshino, S., Wakimoto, T., Onaka, H. \& Abe, I. Chojalactones A-C, cytotoxic butanolides isolated from Streptomyces sp. cultivated with mycolic acid containing bacterium. Org Lett 17, 1501-1504, doi:10.1021/acs.orglett.5b00385 (2015).

16. Hoshino, S. et al. Niizalactams A-C, Multicyclic Macrolactams Isolated from Combined Culture of Streptomyces with Mycolic Acid-Containing Bacterium. J Nat Prod 78, 3011-3017, doi:10.1021/acs.jnatprod.5b00804 (2015).

17. Igarashi, Y. et al. Alchivemycin A, a bioactive polycyclic polyketide with an unprecedented skeleton from Streptomyces sp. Org Lett 12, 3402-3405, doi:10.1021/ol1012982 (2010).

18. Ozaki, T. et al. Identification of the common biosynthetic gene cluster for both antimicrobial streptoaminals and antifungal 5-alkyl-1,2,3,4-tetrahydroquinolines. Org Biomol Chem 17, 2370-2378, doi:10.1039/c8ob02846j (2019).

19. Ozaki, T. et al. Insights into the Biosynthesis of Dehydroalanines in Goadsporin. Chembiochem 17, 218-223, doi:10.1002/cbic.201500541 (2016).

20. Onaka, H. et al. Mycolic acid-containing bacteria activate heterologous secondary metabolite expression in Streptomyces lividans. J Antibiot (Tokyo) 68, 594-597, doi:10.1038/ja.2015.31 (2015).

21. Asamizu, S., Ozaki, T., Teramoto, K., Satoh, K. \& Onaka, H. Killing of Mycolic Acid-Containing Bacteria Aborted Induction of Antibiotic Production by Streptomyces in Combined-Culture. Plos One 10, e0142372, doi:10.1371/journal.pone.0142372 (2015).

22. Ikehata, H. \& Ono, T. The Mechanisms of UV Mutagenesis. J Radiat Res 52, 115-125, doi:10.1269/jrr.10175 (2011).

23. Glickman, B. W., Schaaper, R. M., Haseltine, W. A., Dunn, R. L. \& Brash, D. E. The C-C (6-4) Uv Photoproduct Is Mutagenic in Escherichia-Coli. P Natl Acad Sci USA 83, 6945-6949, doi:DOI 10.1073/pnas.83.18.6945 (1986).

24. Poetsch, A. R. The genomics of oxidative DNA damage, repair, and resulting mutagenesis. Comput Struct Biotec 18, 207-219, doi:10.1016/j.csbj.2019.12.013 (2020).

25. Dizdaroglu, M. \& Jaruga, P. Mechanisms of free radical-induced damage to DNA. Free Radical Res 46, 382-419, doi:10.3109/10715762.2011.653969 (2012).

26. Ohnishi, J., Mizoguchi, H., Takeno, S. \& Ikeda, M. Characterization of mutations induced by N-methyl$\mathrm{N}$ '-nitro-N-nitrosoguanidine in an industrial Corynebacterium glutamicum strain. Mutat Res-Gen Tox 
En 649, 239-244, doi:10.1016/j.mrgentox.2007.10.003 (2008).

27. Tanaka, A., Shikazono, N. \& Hase, Y. Studies on Biological Effects of Ion Beams on Lethality, Molecular Nature of Mutation, Mutation Rate, and Spectrum of Mutation Phenotype for Mutation Breeding in Higher Plants. J Radiat Res 51, 223-233, doi:10.1269/jrr.09143 (2010).

28. Hu, W., Li, W. \& Chen, J. Recent advances of microbial breeding via heavy-ion mutagenesis at IMP. Lett App/ Microbio/ 65, 274-280, doi:10.1111/lam.12780 (2017).

29. Satoh, K. \& Oono, Y. Studies on Application of Ion Beam Breeding to Industrial Microorganisms at TIARA. Quantum Beam Sci 3, doi:ARTN 11 3390/qubs3020011 (2019).

30. Song, Z. Q. \& Luo, L. F. Escherichia coli mutants induced by multi-ion irradiation. J Radiat Res 53, 854-859, doi:10.1093/jrr/rrs061 (2012).

31. Hase, Y. et al. Proton-Cluster-Beam Lethality and Mutagenicity in Bacillus subtilis Spores. Quantum Beam Sci 5, doi:ARTN 25 3390/qubs5030025 (2021).

32. Matuo, Y., Izumi, Y., Furusawa, Y. \& Shimizu, K. Biological effects of carbon ion beams with various LETs on budding yeast Saccharomyces cerevisiae. Mutat Res-Fund Mol M 810, 45-51, doi:10.1016/j.mrfmmm.2017.10.003 (2018).

33. Liu, L. et al. Heavy-ion mutagenesis significantly enhances enduracidin production by Streptomyces fungicidicus. Eng Life Sci 19, 112-120, doi:10.1002/elsc.201800109 (2019).

34. Wang, S. Y. et al. Effects of heavy-ion beam irradiation on avermectin B1a and its analogues production by Streptomyces avermitilis. Eng Life Sci 18, 711-720, doi:10.1002/elsc.201800094 (2018).

35. Wang, S. Y. et al. Significance of Heavy-Ion Beam Irradiation-Induced Avermectin B1a Production by Engineered Streptomyces avermitilis. Biomed Res Int 2017, doi:Artn 5373262 1155/2017/5373262 (2017).

36. Bentley, S. D. et al. Complete genome sequence of the model actinomycete Streptomyces coelicolor A3(2). Nature 417, 141-147, doi:DOI 10.1038/417141a (2002).

37. Blin, K. et al. antiSMASH 6.0: improving cluster detection and comparison capabilities. Nucleic Acids Res 49, W29-W35, doi:10.1093/nar/gkab335 (2021).

38. Chater, K. F., Biro, S., Lee, K. J., Palmer, T. \& Schrempf, H. The complex extracellular biology of Streptomyces. Fems Microbiology Reviews 34, 171-198, doi:10.1111/j.1574-6976.2009.00206.x (2010).

39. Flardh, K. \& Buttner, M. J. Streptomyces morphogenetics: dissecting differentiation in a filamentous bacterium. Nat Rev Microbiol 7, 36-49, doi:10.1038/nrmicro1968 (2009).

40. Sevcikova, B. \& Kormanec, J. Differential production of two antibiotics of Streptomyces coelicolor A3(2), actinorhodin and undecylprodigiosin, upon salt stress conditions. Arch Microbio/ 181, 384389, doi:10.1007/s00203-004-0669-1 (2004).

41. Ou, X. J. et al. SarA influences the sporulation and secondary metabolism in Streptomyces coelicolor M145. Acta Bioch Bioph Sin 40, 877-882, doi:10.1111/j.1745-7270.2008.00466.x (2008). 
42. Matsumoto, K., Ueno, M., Shoji, Y. \& Nakanishi, I. Heavy-ion beam-induced reactive oxygen species and redox reactions. Free Radical Res 55, 450-460, doi:10.1080/10715762.2021.1899171 (2021).

43. Hoff, G., Bertrand, C., Piotrowski, E., Thibessard, A. \& Leblond, P. Genome plasticity is governed by double strand break DNA repair in Streptomyces. Sci Rep-Uk 8, doi:ARTN 5272 1038/s41598-01823622-w (2018).

44. Xu, Z., Li, Y. Y., Wang, Y. M., Deng, Z. X. \& Tao, M. F. Genome-Wide Mutagenesis Links Multiple Metabolic Pathways with Actinorhodin Production in Streptomyces coelicolor. App/ Environ Microb 85, doi:ARTN e03005-18 1128/AEM.03005-18 (2019).

45. Xu, Z. et al. Large-Scale Transposition Mutagenesis of Streptomyces coelicolor Identifies Hundreds of Genes Influencing Antibiotic Biosynthesis. Appl Environ Microb 83, doi:10.1128/Aem.02889-16 (2017).

46. Gunka, K. \& Commichau, F. M. Control of glutamate homeostasis in Bacillus subtilis: a complex interplay between ammonium assimilation, glutamate biosynthesis and degradation. Molecular Microbiology 85, 213-224, doi:10.1111/j.1365-2958.2012.08105.x (2012).

47. Williamson, N. R., Fineran, P. C., Leeper, F. J. \& Salmond, G. P. C. The biosynthesis and regulation of bacterial prodiginines. Nat Rev Microbio/ 4, 887-899, doi:10.1038/nrmicro1531 (2006).

48. Yamamoto, H. et al. EF-G and EF4: translocation and back-translocation on the bacterial ribosome. Nat Rev Microbiol 12, 89-100, doi:10.1038/nrmicro3176 (2014).

49. Kieser, T., Bibb, M. J., Buttner, M. J., Chater, K. F. \& Hopwood, D. A. Practical Streptomyces Genetics. (John Innes Foundation, 2000).

50. Koren, S. et al. Canu: scalable and accurate long-read assembly via adaptive k-mer weighting and repeat separation. Genome Res 27, 722-736, doi:10.1101/gr.215087.116 (2017).

51. Brettin, T. et al. RASTtk: A modular and extensible implementation of the RAST algorithm for building custom annotation pipelines and annotating batches of genomes. Sci Rep-Uk 5, doi:ARTN 8365 1038/srep08365 (2015).

52. Noguchi, H., Taniguchi, T. \& Itoh, T. MetaGeneAnnotator: Detecting Species-Specific Patterns of Ribosomal Binding Site for Precise Gene Prediction in Anonymous Prokaryotic and Phage Genomes. DNA Res 15, 387-396, doi:10.1093/dnares/dsn027 (2008).

53. Laslett, D. \& Canback, B. ARAGORN, a program to detect tRNA genes and tmRNA genes in nucleotide sequences. Nucleic Acids Res 32, 11-16, doi:10.1093/nar/gkh152 (2004).

54. Onaka, H., Taniguchi, S., Ikeda, H., Igarashi, Y. \& Furumai, T. pTOYAMAcos, pTYM18, and pTYM19, actinomycete Escherichia coli integrating vectors for heterologous gene expression. J Antibiot 56, 950-956, doi:DOI 10.7164/antibiotics.56.950 (2003).

\section{Tables}

Table 1, 2 and 3 is available in the Supplemental Files section. 
Figures

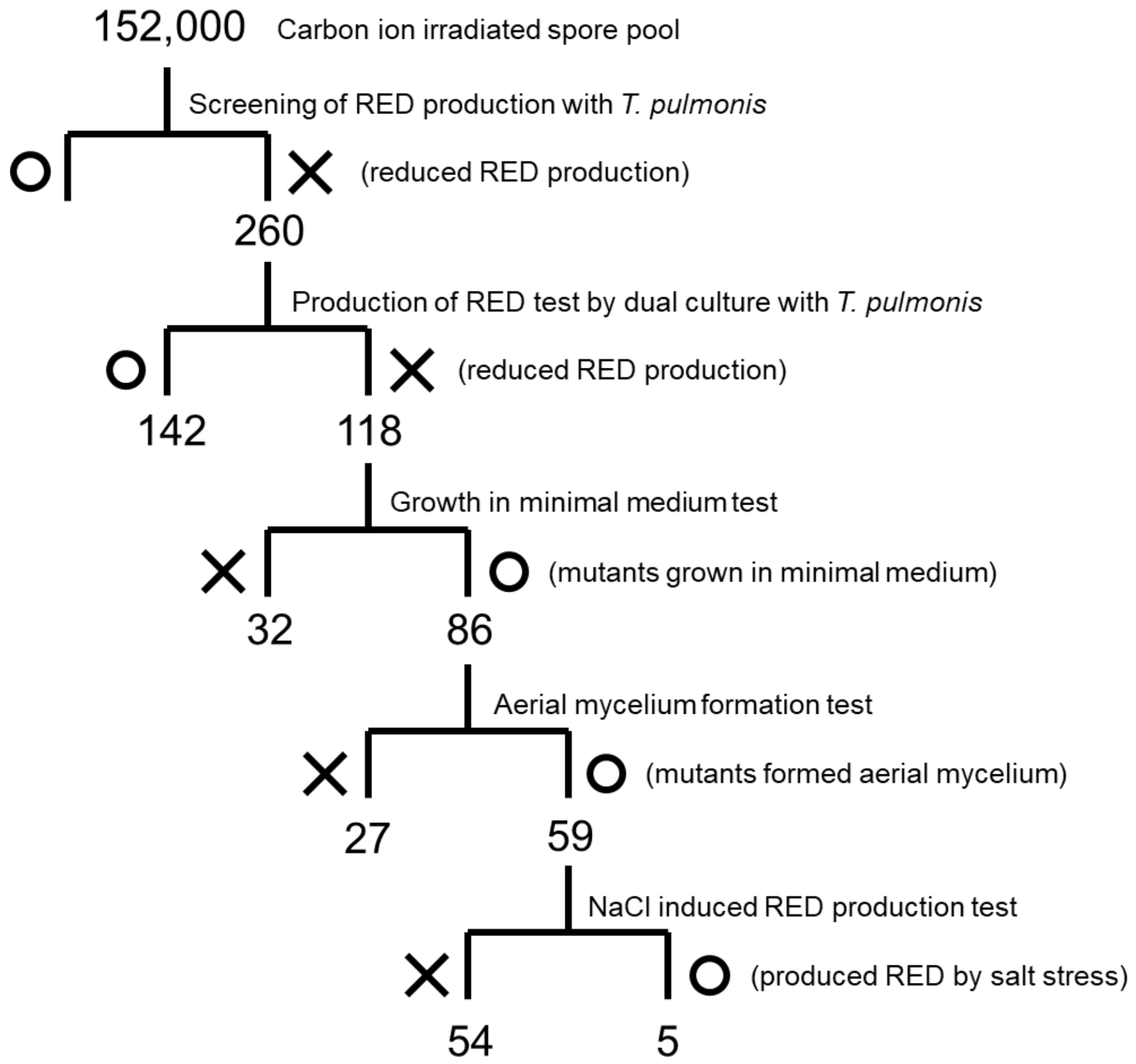

Figure 1

Screening of RED-deficient mutants of S. coelicolor JCM4020. 




Figure 2

Phenotype of the mutants. A: Competitive dual culture among strain JCM4020 wild-type (WT) or Mutant with T. pulmonis. B: Formation of aerial mycelium. C: Production of RED under salt stress condition. 


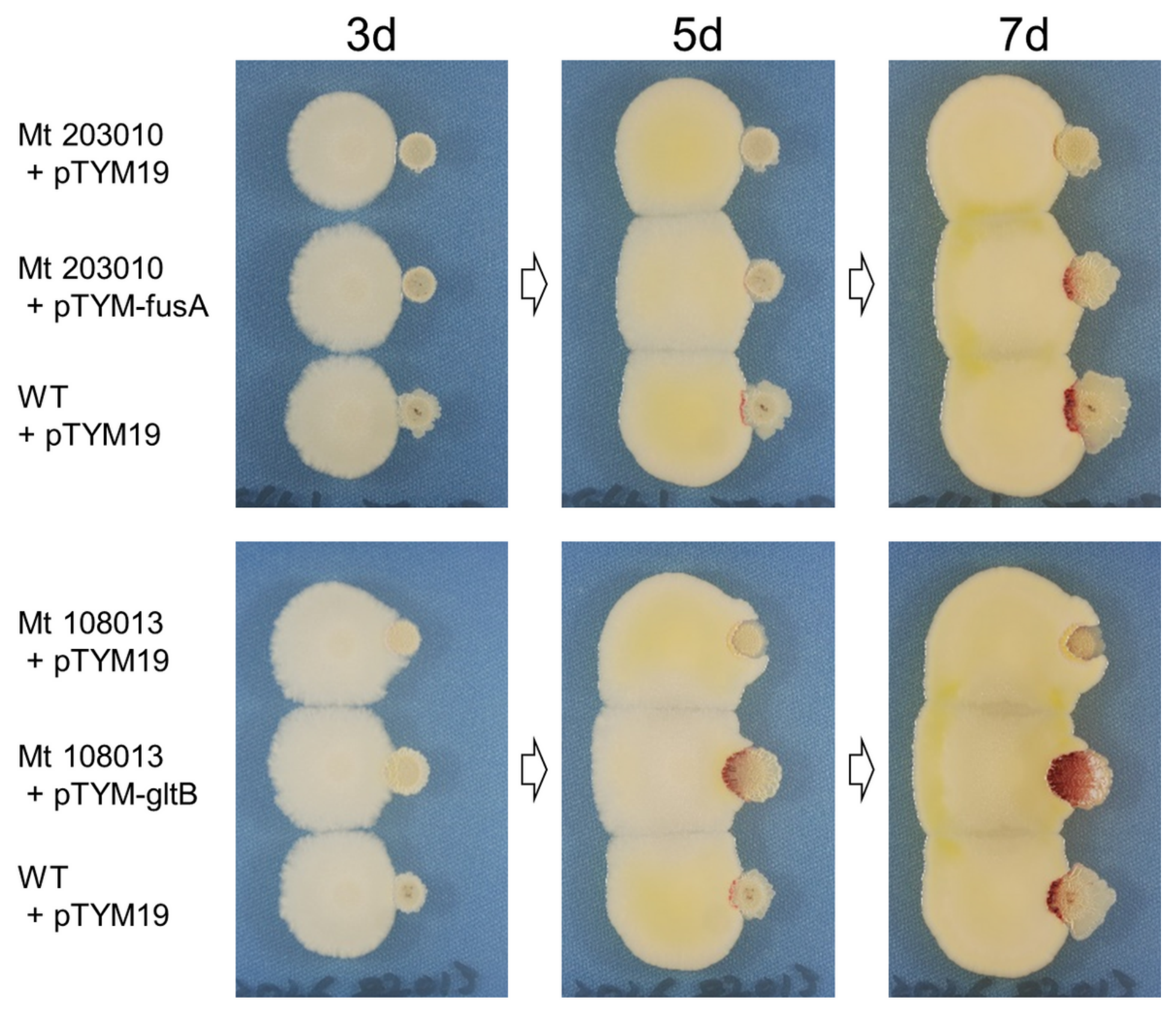

Figure 3

Gene complementations of $g / t B$ in Mt 108013 and fusA in Mt 203010 rescued the productivity of RED in condition interacting with T. pulmonis.

\section{Supplementary Files}

This is a list of supplementary files associated with this preprint. Click to download.

- Yanagisawaetal.SciRepSupplementalFile1.xlsx

- Yanagisawaetal.SciRepSupplementalFiguresandTables.docx

- Yanagisawaetal.SciRepTable1.docx 
- Yanagisawaetal.SciRepTable2.docx

- Yanagisawaetal.SciRepTable3.docx 